\title{
The genome sequence of the large white, Pieris brassicae
}

\section{(Linnaeus, 1758) [version 1; peer review: 2 approved]}

\author{
Konrad Lohse ${ }^{101}$, Alexander Mackintosh1, \\ Darwin Tree of Life Barcoding collective, \\ Wellcome Sanger Institute Tree of Life programme, \\ Wellcome Sanger Institute Scientific Operations: DNA Pipelines collective, \\ Tree of Life Core Informatics collective, Darwin Tree of Life Consortium
}

${ }^{1}$ Institute of Evolutionary Biology, University of Edinburgh, Edinburgh, UK

\begin{tabular}{l}
\hline F1 First published: 12 Oct 2021, 6:262 \\
https://doi.org/10.12688/wellcomeopenres.17274.1 \\
Latest published: 12 Oct 2021, 6:262 \\
https://doi.org/10.12688/wellcomeopenres.17274.1 \\
\hline
\end{tabular}

\section{Abstract}

We present a genome assembly from an individual female Pieris brassicae (the large white; Arthropoda; Insecta; Lepidoptera; Pieridae). The genome sequence is 292 megabases in span. The majority of the assembly is scaffolded into 16 chromosomal pseudomolecules, with the $W$ and $Z$ sex chromosome assembled. Gene annotation of this assembly on Ensembl has identified 12,229 protein coding genes.

\section{Keywords}

Pieris brassicae, large white, genome sequence, chromosomal

This article is included in the Tree of Life gateway.

Open Peer Review
Approval Status
version 1
12 Oct 2021
Norwich, UK Christopher W Wheat
University, Stockholm, Sweden
Any reports and responses or comments on the
article can be found at the end of the article.


Corresponding author: Darwin Tree of Life Consortium (mark.blaxter@sanger.ac.uk)

Author roles: Lohse K: Investigation, Resources, Writing - Original Draft Preparation, Writing - Review \& Editing; Mackintosh A: Writing - Original Draft Preparation, Writing - Review \& Editing;

Competing interests: No competing interests were disclosed.

Grant information: This work was supported by Wellcome through core funding to the Wellcome Sanger Institute (206194) and the Darwin Tree of Life Discretionary Award (218328). KL is supported by a NERC fellowship (NE/L011522/1) and an ERC grant (ModelGenom Land 757648). AM is supported by a E4 PhD studentship.

The funders had no role in study design, data collection and analysis, decision to publish, or preparation of the manuscript.

Copyright: (c) 2021 Lohse $\mathrm{K}$ et al. This is an open access article distributed under the terms of the Creative Commons Attribution License, which permits unrestricted use, distribution, and reproduction in any medium, provided the original work is properly cited.

How to cite this article: Lohse K, Mackintosh A, Darwin Tree of Life Barcoding collective et al. The genome sequence of the large white, Pieris brassicae (Linnaeus, 1758) [version 1; peer review: 2 approved] Wellcome Open Research 2021, 6:262

https://doi.org/10.12688/wellcomeopenres.17274.1

First published: 12 Oct 2021, 6:262 https://doi.org/10.12688/wellcomeopenres.17274.1 


\section{Species taxonomy}

Eukaryota; Metazoa; Ecdysozoa; Arthropoda; Hexapoda; Insecta; Pterygota; Neoptera; Endopterygota; Lepidoptera; Glossata; Ditrysia; Papilionoidea; Pieridae; Pierinae; Pieris; Pieris brassicae (Linnaeus, 1758) (NCBI:txid7116).

\section{Introduction}

The large white, Pieris brassicae, is a Palearctic butterfly species that is common in Europe, North Africa, and Asia. $P$. brassicae larvae typically feed on Brassicaceae species, including cultivated species with agricultural importance such as Brassica oleracea. It has been unintentionally introduced to New Zealand, Chile, and South Africa, although it was later eradicated from New Zealand in 2016 (Phillips et al., 2020). It overwinters as a pupa and is multivoltine. While $P$. brassicae has been listed as Least Concern in the IUCN Red List
(Europe), the Madeiran large white, P. wollastoni (previously considered a subspecies of $P$. brassicae), has not been observed since 1986 and is possibly extinct (IUCN, 2009). P. brassicae has 15 pairs of chromosomes with the female being heterogametic (Bigger, 1975). This karyotype is unusual, as species in the genus Pieris typically possess between 24 and 28 pairs of chromosomes (Robinson, 1971). Its genome size has been estimated with flow cytometry at approximately $260 \mathrm{Mb}$ (Mackintosh et al., 2019).

\section{Genome sequence report}

The genome was sequenced from a single female $P$. brassicae (ilPieBrab1) collected from East Linton, Scotland (latitude 55.977161, longitude -2.667545); Hi-C data were generated from a male $P$. brassicae (ilPieBrab3) collected from the same location (Figure 1). A total of 92-fold coverage in Pacific
A

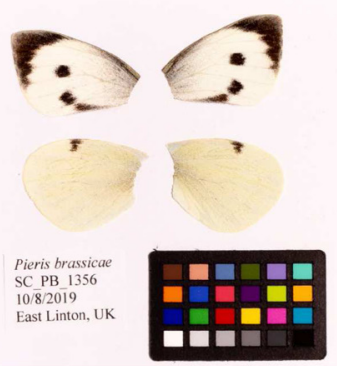

C

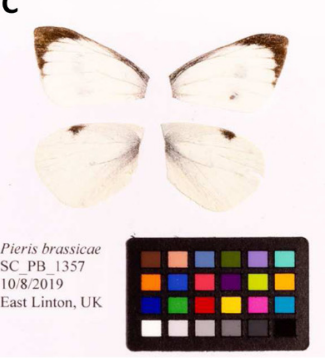

E

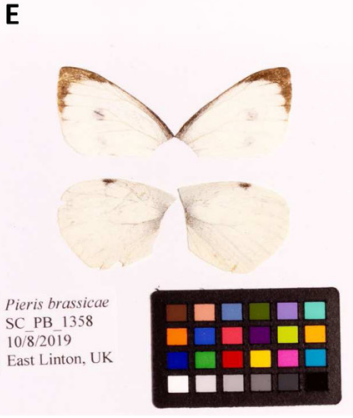

B
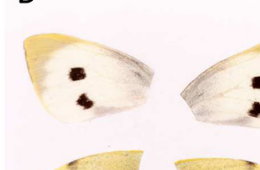

$+2$

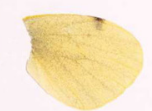

Pieris brassicae ${ }_{1082019}^{C P B} 1356$ East Linton, UK

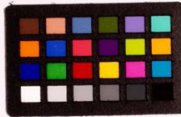

D

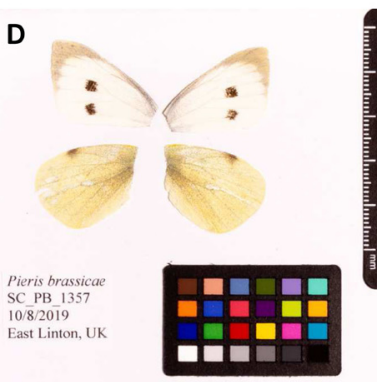

$\mathrm{F}$

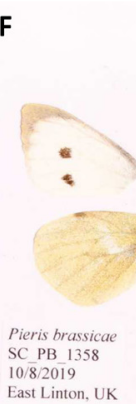

East Linton, $10 \mathrm{~K}$
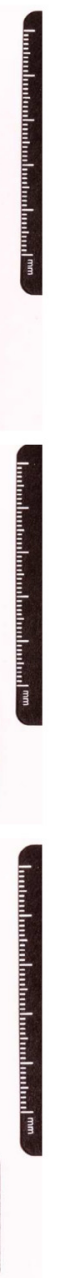

Figure 1. Fore and hind wings of Pieris brassicae specimens from which the genome was sequenced. (A) Dorsal surface view of wings from specimen SC_PB_1356 (ilPieBrab1) from East Linton, used to generate Pacific Biosciences and 10X genomics data. (B) Ventral surface view of wings from specimen SC_PB_1356 (ilPieBrab1) from East Linton, used to generate Pacific Biosciences and 10X genomics data. (C) Dorsal surface view of wings from specimen SC_PB_1357 (ilPieBrab2) from East Linton, used to generate RNASeq data. (B) Ventral surface view of wings from specimen SC_PB_1357 (ilPieBrab2) from East Linton, used to generate RNASeq data. (A) Dorsal surface view of wings from specimen SC_PB_1358 (ilPieBrab3) from East Linton, used to generate Hi-C data. (B) Ventral surface view of wings from specimen SC_PB_1358 (ilPieBrab3) from East Linton, used to generate Hi-C data. 
Biosciences single-molecule long reads (N50 $14 \mathrm{~kb})$ and 138 -fold coverage in 10X Genomics read clouds were generated. Primary assembly contigs were scaffolded with chromosome conformation $\mathrm{Hi}-\mathrm{C}$ data, which was obtained from a different individual (ilPieBrab3). Manual assembly curation corrected 25 missing joins, reducing the scaffold number by $5.87 \%$, and increasing the scaffold $\mathrm{N} 50$ by $1.21 \%$.

The final assembly has a total length of $292 \mathrm{Mb}$ in 402 sequence scaffolds with a scaffold N50 of $22 \mathrm{Mb}$ (Table 1). Of the assembly sequence, $95.46 \%$ was assigned to 16 chromosomal-level scaffolds, representing 14 autosomes (numbered by sequence length), and the $\mathrm{W}$ and $\mathrm{Z}$ sex chromosome (Figure 2-Figure 5; Table 2). The $\mathrm{W}$ chromosome is fragmented as the assembly was scaffolded to an individual of a different sex (ilPieBrab3). The assembly has a BUSCO (Simão et al., 2015) completeness of $99.0 \%$ using the lepidoptera_odb10 reference set. While not fully phased, the assembly deposited is of one haplotype. Contigs corresponding to the second haplotype have also been deposited.

BlobToolKit blob and cumulative sequence plots show that the $\mathrm{W}$ chromosome has regions with microsporidian origin (Figure 3, Figure 4). However, these regions in the read sets are short, do not match across the rest of the scaffold and do not contain any contigs with microsporidian ribosomal subunits. This indicates that this feature is unlikely to be contamination and is more likely to be the result of integration of microsporidian sequence into the genome.

\section{Gene annotation}

The Ensembl gene annotation system (Aken et al., 2016) was used to generate annotation for the Pieris brassicae assembly (GCA_905147105.1, see https://rapid.ensembl.org/ Pieris_brassicae_GCA_905147105.1/; Table 1). The annotation was created primarily through alignment of transcriptomic data to the genome, with gap filling via protein-to-genome alignments of a select set of proteins from UniProt (UniProt Consortium, 2019) and OrthoDB (Kriventseva et al., 2008). Prediction tools, CPC2 (Kang et al., 2017) and RNAsamba (Camargo et al., 2020), were used to aid determination of protein coding genes.

\section{Methods}

Sample acquisition and nucleic acid extraction

A female (ilPieBrab1) and two male (ilPieBrab2, ilPieBrab3) $P$. brassicae (Figure 1) were collected from East Linton, Scotland (latitude 55.977161, longitude -2.667545) using a net by Konrad Lohse, University of Edinburgh, who also identified the samples. The samples were snap-frozen in liquid nitrogen from live.

DNA was extracted from the whole organism of ilPieBrab1 at the Wellcome Sanger Institute (WSI) Scientific Operations core from the whole organism using the Qiagen MagAttract
Table 1. Genome data for Pieris brassicae, ilPieBrab1.1.

\begin{tabular}{|c|c|}
\hline \multicolumn{2}{|l|}{ Project accession data } \\
\hline Assembly identifier & ilPieBrab1.1 \\
\hline Species & Pieris brassicae \\
\hline Specimen & $\begin{array}{l}\text { ilPieBrab1 (genome assembly), } \\
\text { ilPieBrab2 (RNA-Seq), } \\
\text { ilPieBrab3 (Hi-C) }\end{array}$ \\
\hline NCBI taxonomy ID & NCBI:txid7116 \\
\hline BioProject & PRJEB41887 \\
\hline BioSample ID & SAMEA7532735 \\
\hline \multirow[t]{2}{*}{ Isolate information } & $\begin{array}{l}\text { Female, whole organism } \\
\text { (ilPieBrab1) }\end{array}$ \\
\hline & $\begin{array}{l}\text { Males, whole organisms } \\
\text { (ilPieBrab2, ilPieBrab3) }\end{array}$ \\
\hline \multicolumn{2}{|l|}{ Raw data accessions } \\
\hline PacificBiosciences SEQUEL II & ERR6594497 \\
\hline 10X Genomics Illumina & ERR6002738-ERR6002741 \\
\hline Hi-C Illumina & ERR6002742 \\
\hline Illumina PolyA RNA-Seq & ERR6594497 \\
\hline \multicolumn{2}{|l|}{ Genome assembly } \\
\hline Assembly accession & GCA_905147105.1 \\
\hline $\begin{array}{l}\text { Accession of alternate } \\
\text { haplotype }\end{array}$ & GCA_905147085.1 \\
\hline Span (Mb) & 292 \\
\hline Number of contigs & 431 \\
\hline Contig N50 length (Mb) & 21 \\
\hline Number of scaffolds & 402 \\
\hline Scaffold N50 length (Mb) & 22 \\
\hline Longest scaffold (Mb) & 24 \\
\hline BUSCO* genome score & $\begin{array}{l}\text { C:99.0\%[S:98.7\%,D:0.3\%],F:0.3\%, } \\
\text { M:0.7\%,n:5286 }\end{array}$ \\
\hline
\end{tabular}

Gene annotation

\begin{tabular}{l|l}
\begin{tabular}{l|l} 
Number of protein coding \\
genes
\end{tabular} & 12,229 \\
$\begin{array}{l}\text { Average coding sequence } \\
\text { length (bp) }\end{array}$ & 1,736 \\
\hline $\begin{array}{l}\text { Average number of exons } \\
\text { per transcript }\end{array}$ & 10 \\
\hline Average exon size (bp) & 327 \\
\hline Average intron size (bp) & 1687 \\
\hline
\end{tabular}

*BUSCO scores based on the lepidoptera_odb10 BUSCO set using v5.1.2. $\mathrm{C}=$ complete $[\mathrm{S}=$ single copy, $\mathrm{D}=$ duplicated $], \mathrm{F}=$ fragmented, $\mathrm{M}=$ missing, $\mathrm{n}=$ number of orthologues in comparison. A full set of BUSCO scores is available at https://blobtoolkit.genomehubs.org/view/ilPieBrab1.1/dataset/ CAJHUI01/busco. 


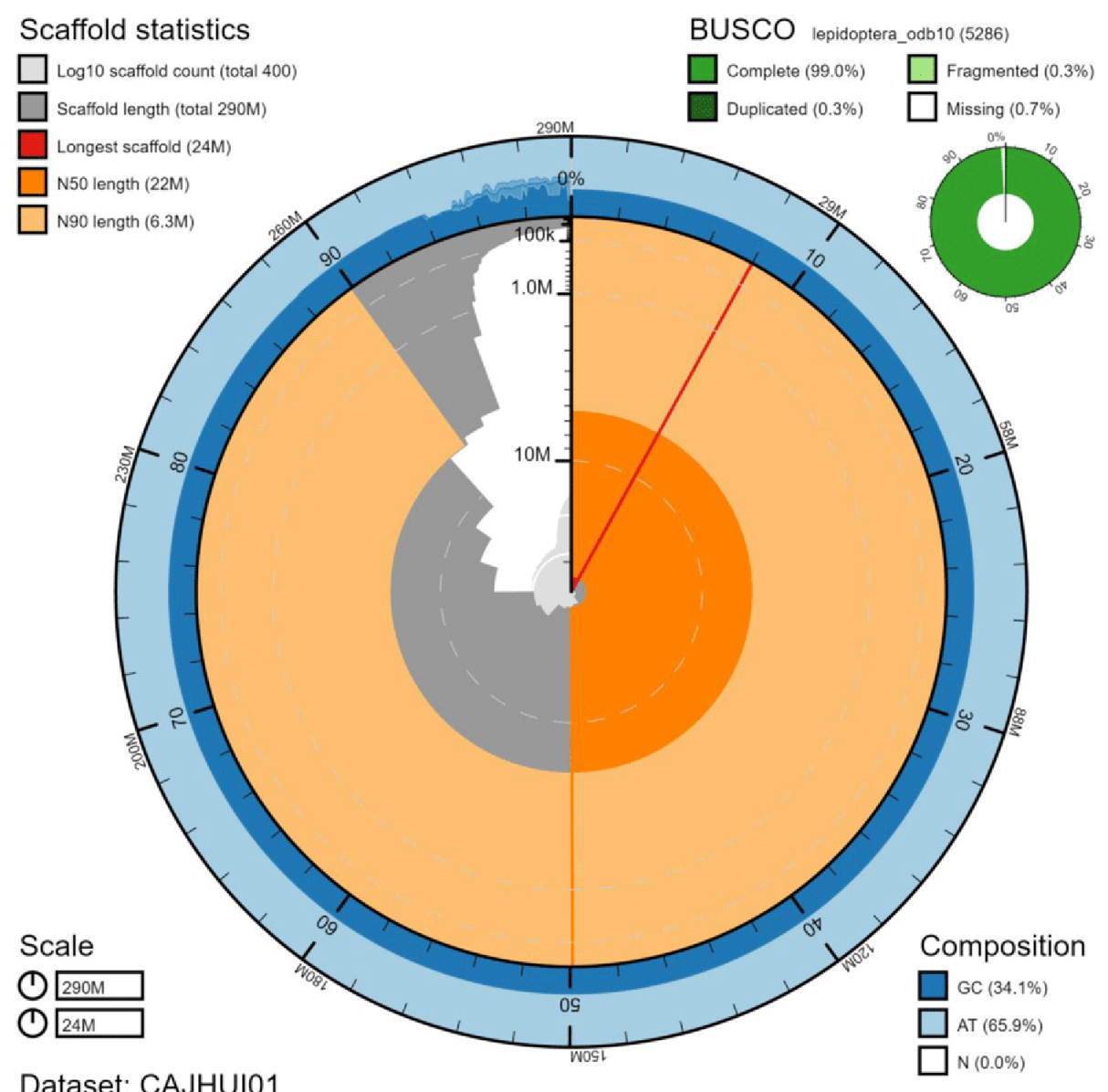

Figure 2. Genome assembly of Pieris brassicae, ilPieBrab1.1: metrics. The BlobToolKit Snailplot shows N50 metrics and BUSCO gene completeness. The main plot is divided into 1,000 size-ordered bins around the circumference with each bin representing $0.1 \%$ of the 292,341,157 bp assembly. The distribution of scaffold lengths is shown in dark grey with the plot radius scaled to the longest chromosome present in the assembly $(23,587,467 \mathrm{bp}$, shown in red). Orange and pale-orange arcs show the N50 and N90 chromosome lengths $(21,549,451$ and 6,322,985 bp), respectively. The pale grey spiral shows the cumulative scaffold count on a log scale with white scale lines showing successive orders of magnitude. The blue and pale-blue area around the outside of the plot shows the distribution of GC, AT and N percentages in the same bins as the inner plot. A summary of complete, fragmented, duplicated and missing BUSCO genes in the lepidoptera_odb10 set is shown in the top right. An interactive version of this figure is available at https://blobtoolkit.genomehubs. org/view/ilPieBrab1.1/dataset/CAJHUI01/snail.

HMW DNA kit, according to the manufacturer's instructions. RNA (also from the whole organism) was extracted from ilPieBrab2 in the Tree of Life Laboratory at the WSI using TRIzol, according to the manufacturer's instructions. RNA was then eluted in $50 \mu \mathrm{l}$ RNAse-free water and its concentration RNA assessed using a Nanodrop spectrophotometer and Qubit Fluorometer using the Qubit RNA Broad-Range (BR) Assay kit. Analysis of the integrity of the RNA was done using Agilent RNA 6000 Pico Kit and Eukaryotic Total RNA assay.

\section{Sequencing}

Pacific Biosciences HiFi circular consensus and 10X Genomics read cloud DNA sequencing libraries were constructed according to the manufacturers' instructions. Poly(A) RNA-Seq libraries were constructed using the NEB Ultra II RNA Library
Prep kit. DNA and RNA sequencing was performed by the Scientific Operations core at the WSI on Pacific Biosciences SEQUEL II (HiFi), Illumina HiSeq $X(10 \mathrm{X})$ and Illumina HiSeq 4000 (RNA-Seq) instruments. Hi-C data were generated from the whole organism of ilPieBrab3 using the Qiagen EpiTect Hi-C kit and sequenced on HiSeq X.

\section{Genome assembly}

Assembly was carried out with HiCanu (Nurk et al., 2020). Haplotypic duplication was identified and removed with purge_dups (Guan et al., 2020). One round of polishing was performed by aligning $10 \mathrm{X}$ Genomics read data to the assembly with longranger align, calling variants with freebayes (Garrison \& Marth, 2012). The assembly was then scaffolded with Hi-C data (Rao et al., 2014) using SALSA2 


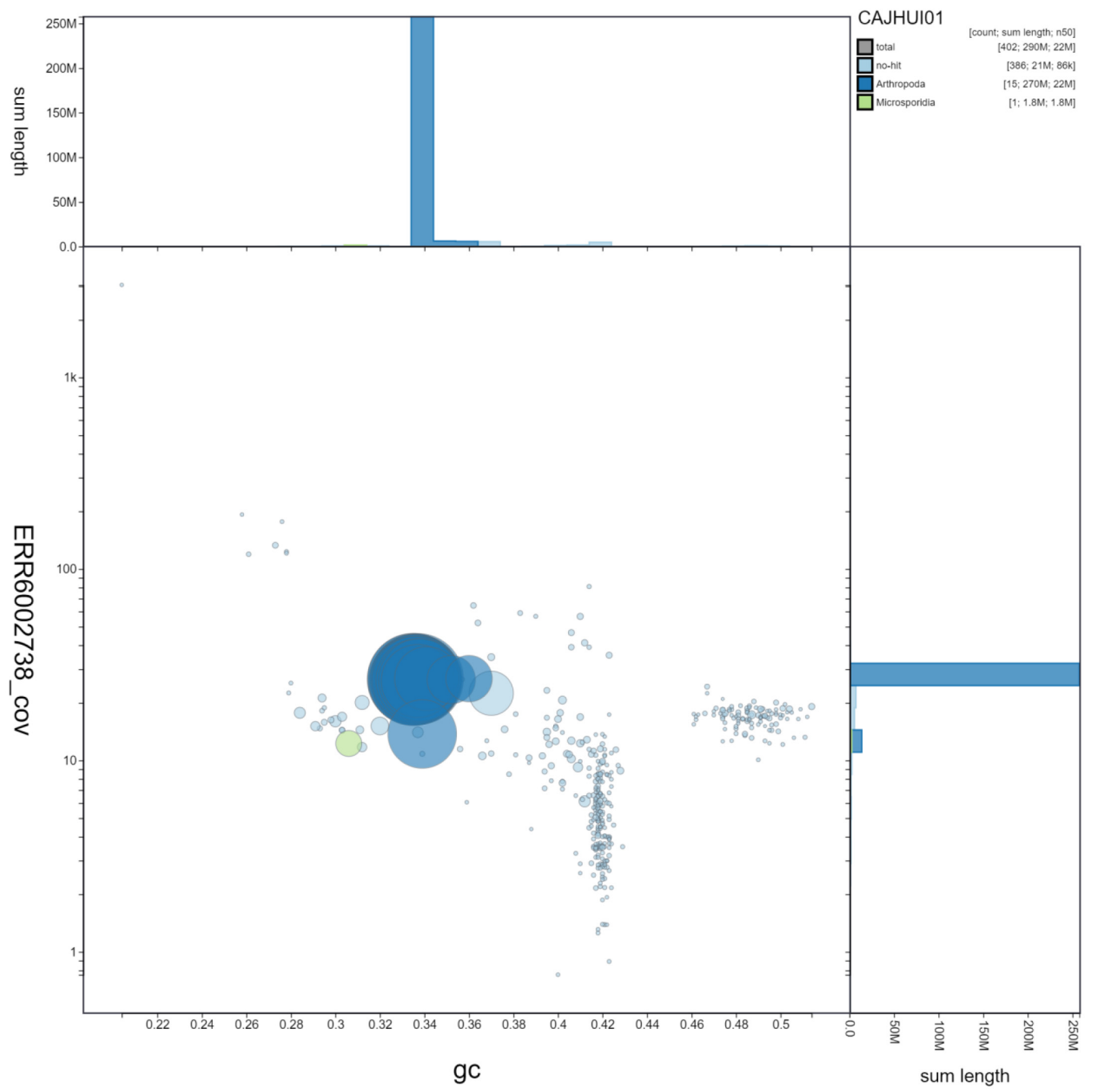

Figure 3. Genome assembly of Pieris brassicae, ilPieBrab1.1: GC coverage. BlobToolKit GC-coverage plot. Scaffolds are coloured by phylum. Circles are sized in proportion to scaffold length. Histograms show the distribution of scaffold length sum along each axis. An interactive version of this figure is available at https://blobtoolkit.genomehubs.org/view/ilPieBrab1.1/dataset/CAJHUI01/blob. 


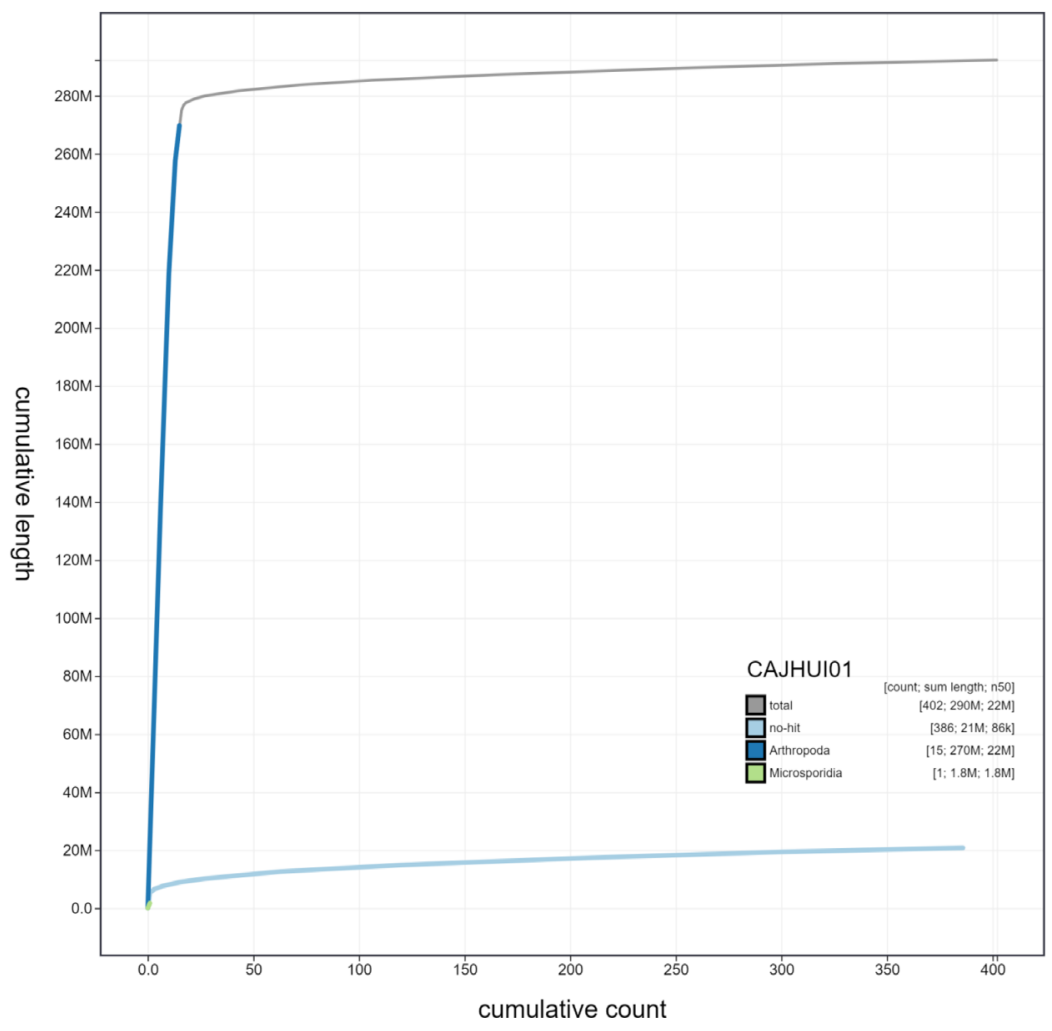

Figure 4. Genome assembly of Pieris brassicae, ilPieBrab1.1: cumulative sequence. BlobToolKit cumulative sequence plot. The grey line shows cumulative length for all scaffolds. Coloured lines show cumulative lengths of scaffolds assigned to each phylum using the buscogenes taxrule. An interactive version of this figure is available at https://blobtoolkit.genomehubs.org/view/ilPieBrab1.1/dataset/ CAJHUI01/cumulative.

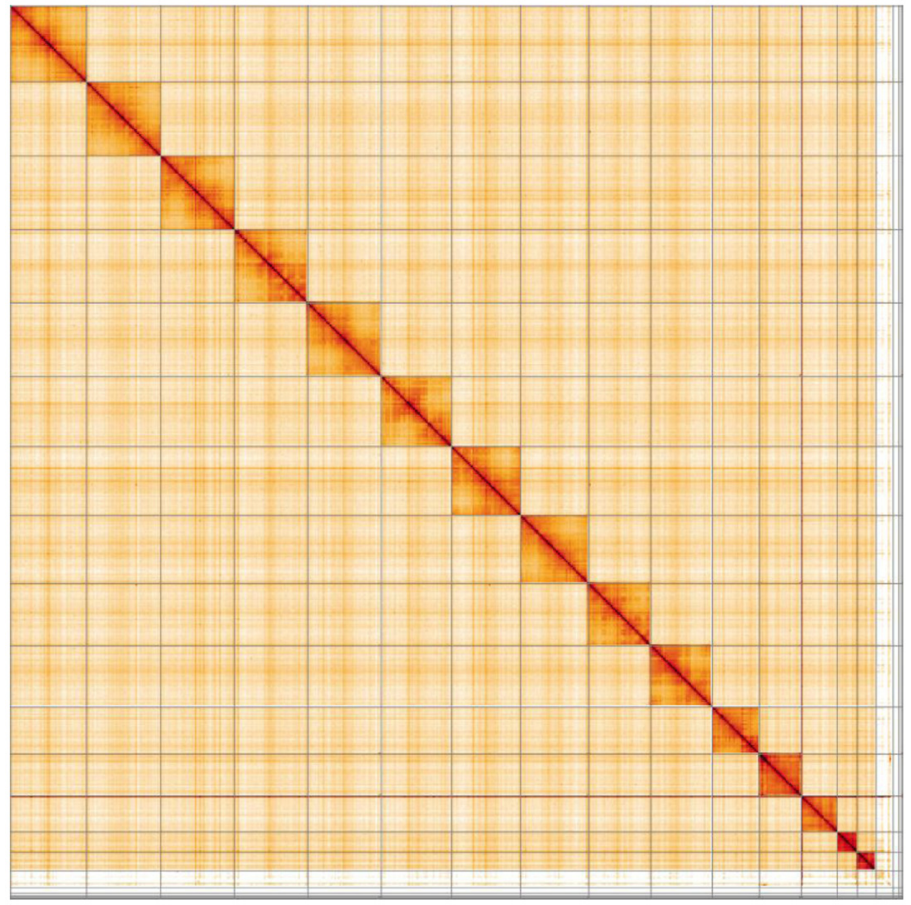

Figure 5. Genome assembly of Pieris brassicae, ilPieBrab1.1: Hi-C contact map. Hi-C contact map of the ilPieBrab1.1 assembly, visualised in HiGlass. Chromosomes are shown in order of size from left to right and top to bottom. 
(Ghurye et al., 2019). The assembly was checked for contamination and corrected using the gEVAL system (Chow et al., 2016) as described previously (Howe et al., 2021). Manual curation was performed using gEVAL, HiGlass (Kerpedjiev et al., 2018) and Pretext. The genome was analysed and BUSCO scores generated within the BlobToolKit environment (Challis et al., 2020). Table 3 contains a list of all software tool versions used, where appropriate.

\section{Table 2. Chromosomal pseudomolecules in the genome assembly of Pieris brassicae, ilPieBrab1.1.}

\begin{tabular}{|c|c|c|c|}
\hline INSDC accession & Chromosome & Size (Mb) & GC\% \\
\hline LR989932.1 & 1 & 23.59 & 33.5 \\
\hline LR989933.1 & 2 & 23.11 & 33.5 \\
\hline LR989934.1 & 3 & 23.07 & 33.7 \\
\hline LR989935.1 & 4 & 22.87 & 33.6 \\
\hline LR989936.1 & 5 & 22.82 & 33.6 \\
\hline LR989937.1 & 6 & 21.88 & 33.5 \\
\hline LR989938.1 & 7 & 21.55 & 33.7 \\
\hline LR989939.1 & 8 & 21.29 & 33.6 \\
\hline LR989940.1 & 9 & 19.44 & 33.7 \\
\hline LR989941.1 & 10 & 19.01 & 33.8 \\
\hline LR989942.1 & 11 & 14.79 & 33.7 \\
\hline LR989944.1 & 12 & 11.23 & 34.1 \\
\hline LR989945.1 & 13 & 6.32 & 35.2 \\
\hline LR989946.1 & 14 & 5.84 & 36 \\
\hline LR989947.1 & W & 5.29 & 37 \\
\hline LR989943.1 & Z & 12.97 & 33.9 \\
\hline LR989948.1 & MT & 0.02 & 20.7 \\
\hline- & Unplaced & 17.25 & 39.1 \\
\hline
\end{tabular}

Table 3. Software tools used.

\begin{tabular}{|l|l|l|}
\hline Software tool & Version & Source \\
\hline HiCanu & 1.0 & Nurk et al., 2020 \\
\hline purge_dups & 1.2 .3 & Guan et al., 2020 \\
\hline SALSA2 & 2.2 & Ghurye et al., 2019 \\
\hline Iongranger align & 2.2 .2 & $\begin{array}{l}\text { https://support.10xgenomics.com/ } \\
\text { genome-exome/software/pipelines/latest/ } \\
\text { advanced/other-pipelines }\end{array}$ \\
\hline freebayes & $1.3 .1-17$-gaa2ace8 & Garrison \& Marth, 2012 \\
\hline gEVAL & N/A & Chow et al., 2016 \\
\hline PretextView & $0.1 . x$ & https://github.com/wtsi-hpag/PretextView \\
\hline HiGlass & 1.11 .6 & Kerpedjiev et al., 2018 \\
\hline BlobToolKit & 2.6 .2 & Challis et al., 2020 \\
\hline
\end{tabular}




\section{Ethical/compliance issues}

The materials that have contributed to this genome note were supplied by a Tree of Life collaborator. The WSI employs a process whereby due diligence is carried out proportionate to the nature of the materials themselves, and the circumstances under which they have been/are to be collected and provided for use. The purpose of this is to address and mitigate any potential legal and/or ethical implications of receipt and use of the materials as part of the research project, and to ensure that in doing so we align with best practice wherever possible.

The overarching areas of consideration are:

- Ethical review of provenance and sourcing of the material;

- Legality of collection, transfer and use (national and international).

Each transfer of samples is undertaken according to a Research Collaboration Agreement or Material Transfer Agreement entered into by the Tree of Life collaborator, Genome Research Limited (operating as the Wellcome Sanger Institute) and in some circumstances other Tree of Life collaborators.

\section{Data availability}

European Nucleotide Archive: Pieris brassicae (large white). Accession number PRJEB42142; https://identifiers.org/ena.embl/ PRJEB42142.
The genome sequence is released openly for reuse. The $P$. brassicae genome sequencing initiative is part of the Darwin Tree of Life (DToL) project. All raw sequence data and the assembly have been deposited in INSDC databases. Raw data and assembly accession identifiers are reported in Table 1.

\section{Acknowledgements}

Members of the Darwin Tree of Life Barcoding collective are listed here: https://doi.org/10.5281/zenodo.4893704.

Members of the Wellcome Sanger Institute Tree of Life programme are listed here: https://doi.org/10.5281/zenodo.5377053.

Members of Wellcome Sanger Institute Scientific Operations: DNA Pipelines collective are listed here: https://doi.org/10.5281/ zenodo.4790456.

Members of the Tree of Life Core Informatics collective are listed here: https://doi.org 10.5281/zenodo.5013542.

Members of the Darwin Tree of Life Consortium are listed here: https://doi.org/10.5281/zenodo.4783559.
Aken BL, Ayling S, Barrell D, et al.: The Ensembl Gene Annotation System. Database (Oxford). 2016; 2016: baw093.

PubMed Abstract | Publisher Full Text | Free Full Text

Bigger TRL: Karyotypes of Some Lepidoptera Chromosomes and Changes in Their Holokinetic Organisation as Revealed by New Cytological Techniques. CYTOLOGIA. 1975; 40(3-4): 713-726. Publisher Full Text

Camargo AP, Sourkov V, Pereira GAG, et al.: RNAsamba: Neural NetworkBased Assessment of the Protein-Coding Potential of RNA Sequences. NAR Genom Bioinform. 2020; 2(1): Iqz024.

PubMed Abstract | Publisher Full Text | Free Full Text

Challis R, Richards E, Rajan J, et al.: BlobToolKit - Interactive Quality Assessment of Genome Assemblies. G3 (Bethesda). 2020; 10(4): 1361-74. PubMed Abstract | Publisher Full Text | Free Full Text

Chow W, Brugger K, Caccamo M, et al.: gEVAL - a web-based browser for evaluating genome assemblies. Bioinformatics. 2016; 32(16): 2508-10. PubMed Abstract | Publisher Full Text | Free Full Text

Garrison E, Marth G: Haplotype-Based Variant Detection from Short-Read Sequencing. arXiv: 1207.3907. 2012.

Reference Source

Ghurye J, Rhie A, Walenz BP, et al.: Integrating Hi-C Links with Assembly Graphs for Chromosome-Scale Assembly. PLoS Comput Biol. 2019; 15(8): e1007273.

PubMed Abstract | Publisher Full Text | Free Full Text

Guan D, McCarthy SA, Wood J, et al.: Identifying and Removing Haplotypic Duplication in Primary Genome Assemblies. Bioinformatics. 2020; 36(9): 2896-98.

PubMed Abstract | Publisher Full Text | Free Full Text

Howe K, Chow W, Collins J, et al.: Significantly Improving the Quality of Genome Assemblies through Curation. GigaScience. 2021; 10(1): giaa153. PubMed Abstract | Publisher Full Text | Free Full Text

IUCN: Pieris Wollastoni. Van Swaay, C., Wynhoff, I., Verovnik, R., Wiemers, M., López Munguira, M., Maes, D., Sasic, M., Verstrael, T., Warren, M. \& Settele, J. IUCN Red List of Threatened Species. IUCN, 2009.

Publisher Full Text
Kang YJ, Yang DC, Kong L, et al: CPC2: A Fast and Accurate Coding Potential Calculator Based on Sequence Intrinsic Features. Nucleic Acids Res. 2017; 45(W1): W12-16.

PubMed Abstract | Publisher Full Text | Free Full Text

Kerpedjiev P, Abdennur N, Lekschas F, et al.: HiGlass: Web-Based Visual Exploration and Analysis of Genome Interaction Maps. Genome Biol. 2018. 19(1): 125.

PubMed Abstract | Publisher Full Text | Free Full Text

Kriventseva EV, Rahman N, Espinosa O, et al.: OrthoDB: The Hierarchical Catalog of Eukaryotic Orthologs. Nucleic Acids Res. 2008; 36(Database issue): D271-75.

PubMed Abstract | Publisher Full Text | Free Full Text

Mackintosh A, Laetsch DR, Hayward A, et al.: The Determinants of Genetic Diversity in Butterflies. Nat Commun. 2019; 10(1): 3466. PubMed Abstract | Publisher Full Text | Free Full Text

Nurk S, Walenz BP, Rhie A, et al.: HiCanu: Accurate Assembly of Segmental Duplications, Satellites, and Allelic Variants from High-Fidelity Long Reads. Genome Res. 2020; 30(9): 1291-1305.

PubMed Abstract | Publisher Full Text | Free Full Text

Phillips CB, Brown K, Green C, et al.: Eradicating the Large White Butterfly from New Zealand Eliminates a Threat to Endemic Brassicaceae. PLoS One. 2020; 15(8): e0236791.

PubMed Abstract | Publisher Full Text | Free Full Text

Rao SS, Huntley MH, Durand NC, et al.: A 3D Map of the Human Genome at Kilobase Resolution Reveals Principles of Chromatin Looping. Cell. 2014; 159(7): 1665-80.

PubMed Abstract | Publisher Full Text | Free Full Text

Robinson R: Lepidoptera Genetics. 1971

Publisher Full Text

Simão FA, Waterhouse RM, Ioannidis P, et al.: BUSCO: Assessing Genome Assembly and Annotation Completeness with Single-Copy Orthologs. Bioinformatics. 2015; 31(19): 3210-12.

PubMed Abstract | Publisher Full Text

UniProt Consortium: UniProt: A Worldwide Hub of Protein Knowledge.

Nucleic Acids Res. 2019; 47(D1): D506-15.

PubMed Abstract | Publisher Full Text | Free Full Text 


\section{Open Peer Review}

\section{Current Peer Review Status:}

\section{Version 1}

Reviewer Report 21 June 2022

https://doi.org/10.21956/wellcomeopenres.19097.r50214

(C) 2022 W Wheat C. This is an open access peer review report distributed under the terms of the Creative Commons Attribution License, which permits unrestricted use, distribution, and reproduction in any medium, provided the original work is properly cited.

\section{Christopher W Wheat}

Department of Zoology, Faculty of Science, Stockholm University, Stockholm, Sweden

This is a standard genome report for a new species, quickly covering the minimal aspects of the species ecology. The vast majority of genomic methods and results are reported clearly and the work is good.

I have only three concerns. First, with regards to the polishing, it is not clear how the polishing was actually carried out (only the mapping and SNP identification are described). Details of how 10X data went through PCR duplication removal, as well as barcode removal, and ultimately how the polishing was actually performed, is never reported. Second, it is not clear how "Manual assembly curation corrected" the genome. An additional sentence here could easily fix this. Third, BUSCO reports only stating "completeness" are really quite useless. Unless people report complete single copy, the level of duplication in the BUSCO result is not known. This needs to be corrected, and more details added to the relevant figure.

Is the rationale for creating the dataset(s) clearly described?

Yes

Are the protocols appropriate and is the work technically sound?

Yes

Are sufficient details of methods and materials provided to allow replication by others? Partly

Are the datasets clearly presented in a useable and accessible format? Yes

Competing Interests: No competing interests were disclosed.

Reviewer Expertise: Butterfly genomics 


\section{I confirm that I have read this submission and believe that I have an appropriate level of expertise to confirm that it is of an acceptable scientific standard.}

Reviewer Report 25 October 2021

https://doi.org/10.21956/wellcomeopenres.19097.r46391

(c) 2021 Nash W. This is an open access peer review report distributed under the terms of the Creative Commons Attribution License, which permits unrestricted use, distribution, and reproduction in any medium, provided the original work is properly cited.

\section{Will Nash}

The Earlham Institute, Norwich, UK

This article describes the assembly and annotation of the genome of Pieris brassicae, the Large White butterfly. Three individuals were used to generate the data contributing to the assembly and annotation: the first contributed high molecular weight DNA which was used for PacBio Hifi and 10X sequencing, the second contributed RNA-seq which was used in the annotation process, the third was used to generate $\mathrm{Hi}-\mathrm{C}$ chromosome conformation data.

The rationale for this project is made clear in a well written introduction which guides the reader to the status of the agricultural importance of the species, as well as its status as an introduced species in several countries.

The protocols described are exemplary and in line with contemporary studies. The sequencing strategy is appropriate for an invertebrate species of the size of $P$. brassicae. The bioinformatic pipeline that is used to assemble the Hifi reads, purge haplotigs and polish with 10X is cutting edge and is married with the use of the SALSA2 software to scaffold with $\mathrm{Hi}$-C. The final assembly is well quality controlled with the use of an appropriate BUSCO database as well as BlobToolKit providing interesting information on the potential integration of microsporidian sequence into the genome.

The genome note is of sufficient detail throughout, but I wonder if it would help reproducibility to include the specific settings used for the bioinformatic tools?

Analyses of datasets generated are presented clearly in large, well coloured figures with informative captions. There are also interactive versions of some figures available. The assembly data is accessible and linked in the manuscript.

Is the rationale for creating the dataset(s) clearly described?

Yes

Are the protocols appropriate and is the work technically sound? Yes

Are sufficient details of methods and materials provided to allow replication by others? 
Partly

Are the datasets clearly presented in a useable and accessible format?

Yes

Competing Interests: No competing interests were disclosed.

Reviewer Expertise: Evolutionary Genomics

I confirm that I have read this submission and believe that I have an appropriate level of expertise to confirm that it is of an acceptable scientific standard. 